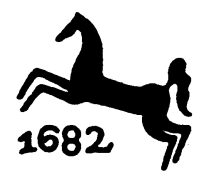

LITERATURBETRIEB IN DER DDR

SCHRIFTSTELLER UND LITERATURINSTANZEN 
REINHILD KÖHLER-HAUSMANN

\section{Literaturbetrieb in der DDR}

Schriftsteller und Literaturinstanzen 
CIP-Kurztitelaufnahme der Deutschen Bibliothek

Köhler-Hausmann, Reinhild:

Literaturbetrieb in der DDR: Schriftsteller u.

Literaturinstanzen / Reinhild Köhler-Hausmann.

- Stuttgart: Metzler, 1984.

(Metzler-Studienausgabe)

ISBN 978-3-476-00554-0

ISBN 978-3-476-00554-0

ISBN 978-3-476-03193-8 (eBook)

DOI 10.1007/978-3-476-03193-8

(C) 1984 Springer-Verlag GmbH Deutschland Ursprünglich erschienen bei J. B. Metzlersche Verlagsbuchhandlung und Carl Ernst Poeschel Verlag GmbH in Stuttgart 1984 


\section{INHALT}

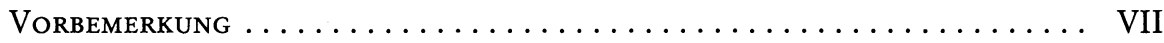

1. Einleitung $\ldots \ldots \ldots \ldots \ldots \ldots \ldots \ldots \ldots \ldots \ldots \ldots \ldots \ldots \ldots \ldots \ldots \ldots \ldots \ldots$

2. Darstellung der Voraussetzungen für die Fragestellung

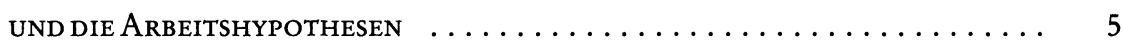

2.1. Bestimmung des Standorts literarischer Arbeit in der $D D R \ldots \ldots \ldots \ldots \ldots$

2.1.1. Lukács Einordnung von "Künstler ", "Parteidichter « und»Partisan" . . . . 5

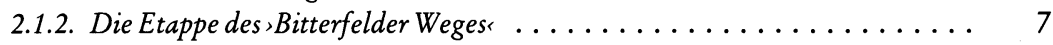

2.1.2.1. Der gescheiterte Versuch einer allseitigen Demokratisierung der Kunst dargestellt an der Aktion des Brigadetagebuchs ........ 9

2.1.3. Das Verbältnis von Literatur und technischer Revolution . . . . . . . . . 12

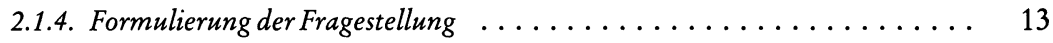

2.2. Zum Selbstverständnis und Funktionsverständnis des Schriftstellers in der DDR . . 14

2.3. Das hermeneutische Verfabren der Arbeit und allgemeine Hypothesen zum bäufigen

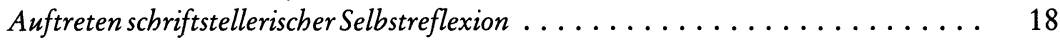

3. Thematisierung von Schriftstellern im literarischen Werk ALS FIKTIVE INTERAKTION MIT $>$ LITERARISCHEN INSTANZEN $<\ldots \ldots \ldots \ldots$

3.1. Aus dem Hochschulbereich: Das Verbältnis von proletarischem Schriftsteller und reflektieren der Intelligenz, dargestellt an Günter de Bruyns» Preisverleibung " . . . . .

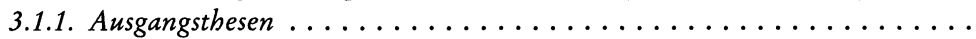

3.1.2. Die Entwicklungsetappen der Protagonisten und ibre veränderte gegenwärtige Position im Hinblick auf ibr Funktionsverständnis von Literatur . . . . .

3.1.3. Thematisierte ,Kulturinstanzen', die bei der Suche nach Bewertungsmaßstä-

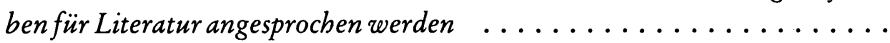

3.1.4. Die Rezeption eines anderen literarischen Werkes, die vermittelte Bezug-

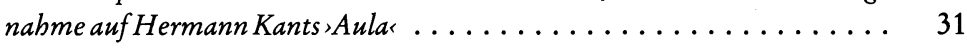

3.1.5. Untersuchungen zur Erzäblstrutkur der»Preisverleibung « . . . . . 36

3.1.6. Das Verbältnis von fiktiver Erzäblperspektive im Roman zu de Bruyns Er-

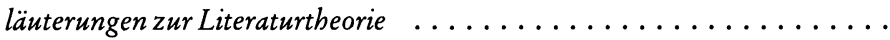

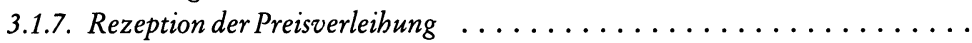

3.2. Aus dem Verlags- und Verwertungsbereich Film: Das Verbältnis von Schriftsteller und, Verteilerinstanz‘, dargestellt an Jurek Beckers `Irrefübrung der Bebörden` . . .

3.2.1. Ausgangsthesen . ......................

3.2.2. Interpretation und Analyse des Stellenwerts der fiktiven, Geschichten, Gregor Bieneks für die Erzäblabsicht von J. Becker . ............ 
3.2.3. Die im Roman gestaltete Wirkung, Reaktion und Stellungnabme zu den schriftstellerischen Werken der Hauptfigur . ...............

3.2.4. Die Rezeption von Jurek Beckers»Irreführung der Bebörden « ........

3.3. Aus der Theaterarbeit: Die spezifische Utopie der Entwicklung eines Stückes vom schriftstellerischen Entwurf zur kreativen Bewältigung im Prozeß der kollektiven Aneignung. Dargestellt an Volker Brauns »Das ungezwungene Leben Kasts" . . . . .

3.3.1. Ausgangsthesen . ......................

3.3.2. Die selektive Verknüpfung von Erfabrungen eines anderen Autoren (Büchners "Lenz «) mit den eigenen, zur Vermittlung seines Lebensgefübls . . . .

3.3.3. Der Bericht „Die Bretter als Plädoyer für eine Integration von Fiktion und Realität beim wirkungsbezogenen Umschreiben einer Vorlage wäbrend der Inszenierungsarbeit . ...................

3.3.4. Die Schreibmotivation Brauns in "Kast « als retrospektiver Legitimationsakt

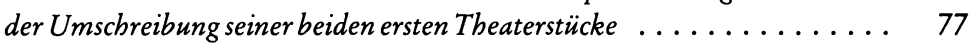

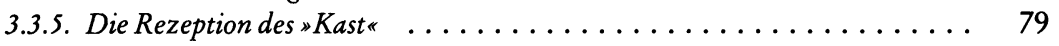

4. Die DDR-Literaturgesellschaft $\ldots \ldots \ldots \ldots \ldots \ldots \ldots \ldots \ldots \ldots$

4.1. Skizzierung der ideologischen Verschiebung nach der Ablösung Ulbrichts durch Honecker

4.2. Die kulturpolitischen Tendenzen der frühen 70er Jabre $\ldots \ldots \ldots \ldots \ldots \ldots$

4.3. Literaturtheoretischer Exkurs zur Rezeptionsästhetik .............

4.4. Rezeptionsbeeinflussung (Stimulierung und Steuerung) . . . . . . . . .

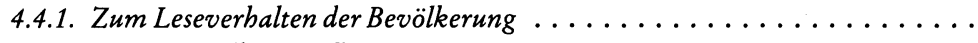

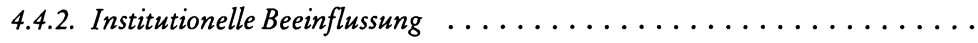

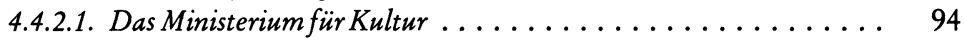

4.4.2.2. Verlagswesen ..................... 98

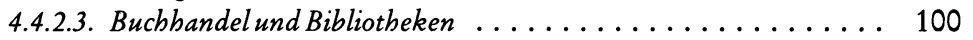

4.4.3. Meinungsbildung durch Literaturinterpretation . . . . . . . . . . 102

4.4.3.1. Das Literaturverständnis der ersten DDR-Literaturgeschichte . . 102

4.4.3.2. Das didaktische Konzept des Literaturunterrichts . . . . . . . 107

4.4.3.3. Kontroversen um die Literaturkritik . . . . . . . . . . . . . . 117

4.4.3.3.1. Exemplarische Untersuchung eines literaturkritischen Vorgangs an Hand von Günter Kunerts »Pamphlet

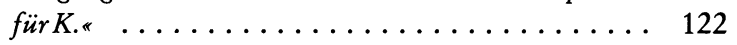

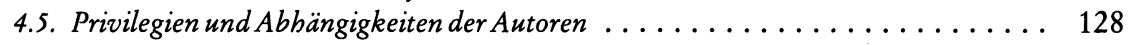

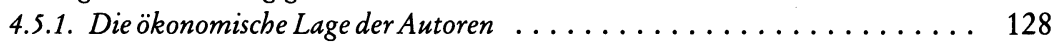

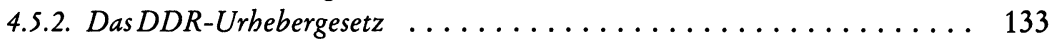

4.5.3. Der Schriftstellerverband der $D D R \ldots \ldots \ldots \ldots \ldots \ldots \ldots \ldots \ldots \ldots$

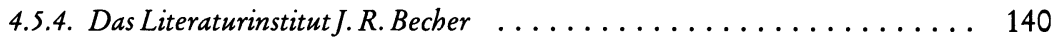

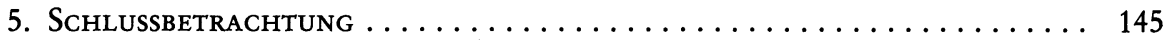

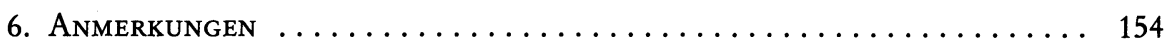

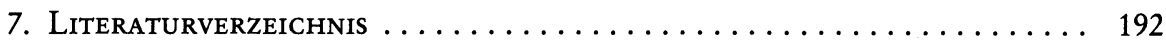




\section{VORBEMERKUNG}

Die vorliegende Arbeit, die zu Beginn der achtziger Jahre DDR-Literaturphänomene beschrieben hat, die sich auf das vorangegangene Jahrzehnt beziehen, unterliegt der Gefahr durch neue Entwicklungen bereits widerlegt oder auch in unfreiwilligem Sarkasmus bestätigt zu werden. Dies um so mehr, zumal es zentrales Anliegen dieser Untersuchung war, sich über ein enger literaturwissenschaftliches Interesse hinaus, kulturpolitischen und gesellschaftlichen Fragen zu nähern und zwar dem schriftstellerischen Selbstbild und Rollenverständnis in der DDR, wie es sich in den ersten zwei Dritteln der siebzigerJahre repräsentiert hat.

Obgleich über zwei Jahre Differenz zwischen dem Redaktionsschluß der Arbeit und ihrer Veröffentlichung liegen, erstaunt dennoch die Verschiebung der kulturellen Strukturen in der DDR. Daß auch eine Anzahl der hier angesprochenen Autoren inzwischen dem unmittelbaren Einfluß des Modells DDR-Sozialismas durch Übersiedlung in den Westen entwichen sind, spricht zwar für die Aktualität des Themas, aber nicht für ein realistisches Handlungspotential von DDR-Künstlern. Die Situation hat sich vielmehr in diesen letzten Jahren so gewandelt, daß neben den beiden DDR-internen, bewußtseinsmäßig divergierenden Autorengruppen, den an genormten Wirklichkeitsvorstellungen festhaltenden Vertretern einerseits und den selbstkritisch reflektierenden Schriftstellern andererseits, eine neuartige, dritte Gruppe entstanden ist, die von der BRD aus - sicherlich anders als frühere DDR-Emigranten - auch weiterhin DDR-Erfahrungen, Wünsche und Ansprüche in ihren Werken aufgreifen und verarbeiten wird.

Abschließend möchte ich an dieser Stelle darauf hinweisen, daß mir Seminare und weiterführende Gespräche mit den Professoren Hillmann, Mandelkow und Emmerich die Anregung und das vertiefte Interesse für die vorliegende Thematik vermittelt haben. Ihnen, sowie der stärker im praktischen und emotionalen Bereich angesiedelten Unterstützung von Dr. H. Lohmann, Dr. J. Eckert, K. Köhler, den Mitarbeiterinnen der Bibliothek des Gesamtdeutschen Instituts in Bonn, sowie den DDR-Autoren, die mir als direkte oder indirekte Gesprächspartner auch weiterhin richtungweisend für eine Utopie vom menschenwürdigen Sozialismus bleiben werden, möchte ich sehr herzlich danken.

Hamburg, im September 1983 
Ich denke wirklich, daß das, was noch nicht $[\ldots]$ beschrieben ist, auch im gesellschaftlichen Bewußtsein noch nicht vorhanden sein kann und dunkel bleibt. Und das sollte Prosa tun: das Unkenntliche erkenntlich, erkennbar machen, das vom Menschen noch nicht Gesehene, ohne Verständnis Erlebte oder noch nicht bis zu Ende Durchdachte sichtbarer, verständlicher machen; ihm möglich machen, es sich anzueignen.

Christa Wolf 